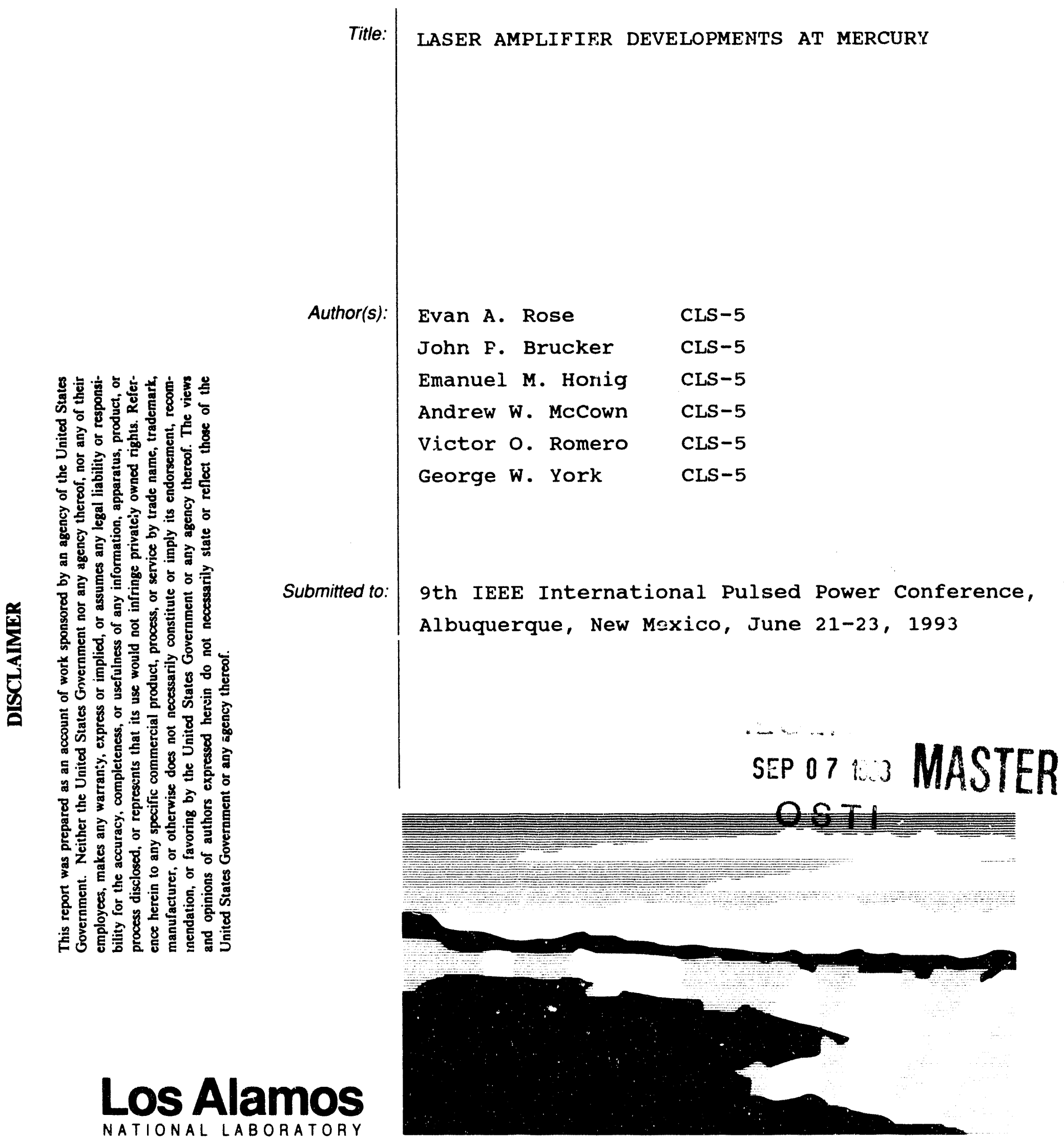

Los Alamos National Laboratory, an aftirmative action/equal opportunity employer, is operated by the University of California for the U.S. Department of Energy under contract W-7405-ENG-36. By acceptance of this article, the publisher recognizes that the U.S. Government retains a nonexclusive, royalty-free license to publish or reproduce the published form of this contribution, or to allow others to do so, for U.S. Government purposes. The Los Alamos National Laboratory requests that the publisher identity this article as work performed under the auspices of the U.S. Department of Energy. 


\section{LASER AMPLIFIER DEVELOPMENTS AT MERCURY}

Evan A. Rose, John P. Brucker, Emanuel M. Honig,

Andrew W. McCown, Victor O. Romero, George W. York

Los Alamos National Laboratory

P.O. Box 1663, Mail Stop E-543

Los Alamos, New Mexico 87545

\section{Abstract}

Electron-beam pumped laser amplifiers have been modified to address the mission of krypton-fluoride excimer laser technology development. Methods are described for improving the performance and reliability of two pre-existing amplifiers at minimal cost and time. Preliminary performance data are presented to support the credibility of the approach.

\section{Introduction}

The Mercury KrF excimer laser system [1] replaces the Aurora $\mathrm{KrF}$ system [2] at Los Alamos. The goal of the Aurora system was to deliver kilojoules of $248 \mathrm{~nm}$ light to a target [3]. The Mercury system explores $\mathrm{KrF}$ laser technology for inertial confinement fusion applications. Mercury will explore bandwidth, pulse shaping, and short-pulse amplification using an operational $\mathrm{KrF}$ laser system, from front end to target.

Mercury incorporates exploration of laser subsystems, including optical, diagnostic, and pulsed power. The pulsed power subsystems have been modified from Aurora hardware. Changes, improvements, and innovations are being incorporated into Mercury. By making incremental changes to working subsystems, this development path saves time and money and improves reliability. New technical ideas are explored on a working system. rather than in isolation.

\section{Mercury Objectives}

Table 1 summarizes some of the principal parameters of the Mercury system in Phase I (current) and Phase II (planned). Extensive documentation of Phase I is available in reference [4].

\begin{tabular}{|lll|}
\hline & Table 1 \\
Mercury Parameter & $\frac{\text { Phase I }}{200 \mathrm{ps}-5 \mathrm{~ns}}$ & $\frac{\text { Phase II }}{200 \mathrm{ps}-5 \mathrm{~ns}}$ \\
pulse width & 120 joules & 800 joules \\
energy on target & 200 microns & 200 microns \\
laser spot size & 24 & 48 \\
laser beam count & & \\
\hline
\end{tabular}

Areas of technical development are summarized in Table 2. The $\mathrm{KrF}$ laser is a promising inertial fusion energy (IFE) driver, since it incorporates shor wavelength $(248 \mathrm{~nm})$, broad bandwidth (100 wave numbers), high dynamic range pulse-shaping, high energy efficiency, and the capability for high-repetition-rate operation. Mercury will address many of these parameters.

\begin{tabular}{|ll|}
\hline Development Area & $\begin{array}{l}\text { Table 2 } \\
\text { Detail } \\
\text { broad bandwidth } \\
\text { pulse shaping } \\
\text { beam smoothing } \\
\text { laser beams }\end{array}$ \\
$\begin{array}{l}\text { gas excitation } \\
\text { laser extraction } \\
\text { amplified spontaneous emission } \\
\text { optical fabrication } \\
\text { integrated diagnostics } \\
\text { efficiency \& reliability }\end{array}$ \\
\hline
\end{tabular}

Los Alamos has developed a suite of codes to model the gas excitation, laser extraction, and amplified spontaneous emission processes. The codes are essential for accurate scaling predictions. We have developed on-site optical fabrication methods, which promise to reduce optics costs. An intr grated diagnostics system is being installed to monitor and vack laser beam properties throughout the system. We are exploring amplifier efficiency and reliability, particularly with regard to the pulsed power systems and electron beam transport from diode to laser medium.

The Mercury facility explores KrF technology issues on a working system, indicating a path for scaling to higher energy systems to meet IFE objectives [5,6,7].

\section{Amplifier Modifications}

Mercury Phase I uses two electron-beam pumped laser amplifiers to achieve greater than $100 \mathrm{~J}$ on target. Aurora's first and fourth amplifiers have been modified to meet Phase I objectives. Aurora's second and third amplifiers were decommissioned. The cost and time for reconfiguration have been reduced compared to building new amplifiers. Reliability has been increased by modification of proven designs to reduce the parts count and to reduce the electrical stresses.

Mercury Amplifier 1 (Al or Charon) achieves an increase in pump power over its previous incarnation, the Aurora Small Aperture Module (SAM), by incorporating a magnetic guide field and a diode-foil structure that protrudes into the laser cavity. Electron pumping is intensified in the extracted volume. The Marx generator capacitance is reduced, achieving a great enhancement in reliability, particularly foil lifetime, with no degradation in pumping.

Mercury Amplifier 2 (A2 or Pluto) is a downsized version of the Aurora Large Aperture Module (LAM). The laser-cavity aperture is reduced from $100 \mathrm{~cm}$ to $40 \mathrm{~cm}$. The laser is pumped from only one side, reducing the pulsed power parts count by two. In addition, the pulse length is reduced by $25 \%$, and the voltage is reduced iy $20 \%$. Improved reliability is anticipated through partscount reduction and electrical-stress reduction. A new foil-support structure is designed to reduce mechanical stress on the foil.

\section{Modifications to Charon (Amplifier 1)}

The first amplifier, Charon, was constructed by modifying Aurora's first amplifier, Small Aperture Module (SAM).

\section{Pulsed Power Reduction}

The SAM amplifier started life as a Maxwell Excitron, pressed into service on Aurora to boost the iaser energy from a Lumonics amplifier. It was nominally a run-down Marx with a peaker. The Marx generator was $229 \mathrm{nF}$ (two 800-nF capacitors in each of seven stages) in series with $600 \mathrm{nH}$ and $2.5 \Omega$. The peaker is $9.5 \mathrm{nF}$ in series with $50 \mathrm{nH}$. A self-breaking output switch connects to an 8- $\Omega$ diode through $300 \mathrm{nH}$. A trigatron diverts the tail of the pulse through $120 \mathrm{nH}$ and $3 \Omega$. The pulsed power's task is to pump the laser amplifier for a $60 \mathrm{~ns}$ laser extraction period.

Figure 1 shows the diode voltage from the pulsed power circuit model (70 kV per stage), including operation of the divertor. The darker trace of Figure 1 shows the Marx generator as it is today, 
reduced to one capacitor per stage. The divertor cuts off the RC decay of the pulse, leaving the peaker signature. The useful pulse is essentially the same for both circuits. but the energy absorbed by the divertor is significantly reduced with the modification.

In actual operation, the new circuit has proven far superior to the original. In the past, when the divertor failed to operate, the pressure foil was destroyed by heating from the long, unclipped tail of the pulse. Now the pressure foil survives. Reliability has been greatly enhanced.

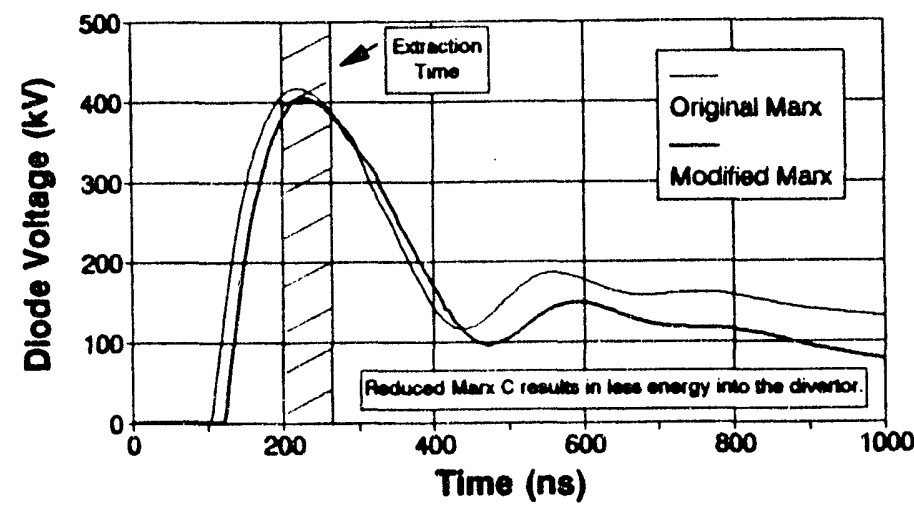

Figure 1. The capacitors are reduced from two per stage on SAM Marx generator to one per stage on Charon. There is litule effect on the diode waveform. Reducing the stored energy puts less stress on the divertor and increases foil lifetime.

\section{Pump Power Increase}

Figure 2 shows the small-signal gain in SAM and in Charon. Small-signal gain is an indicator of the pump power in the laser gas. The Phase I Mercury design calls for $4.5 \% \mathrm{~cm}^{-1}$.

Initially the laser-extracted volume of SAM started approximately $6 \mathrm{~cm}$ from the pressure foil. A 50\% improvement in laser pumping was predicted by extending the foil forward by $4.5 \mathrm{~cm}$ into the laser chamber. This assumes no extra electron beam losses in the increased drift space from anode to foil. Additionally, the pumping would be more uniform across the extracted volume.

There was no magnetic guide field on SAM. A Monte-Carlo electron beam energy deposition calculation (DEP3D) indicated that the pumping would be doubled by adding a $1-\mathrm{kG}$ magnetic guide field and leaving the extracted volume in the same location. The decrease in electron scattering out of the extracted volume (upward and downward) increases the pumping.

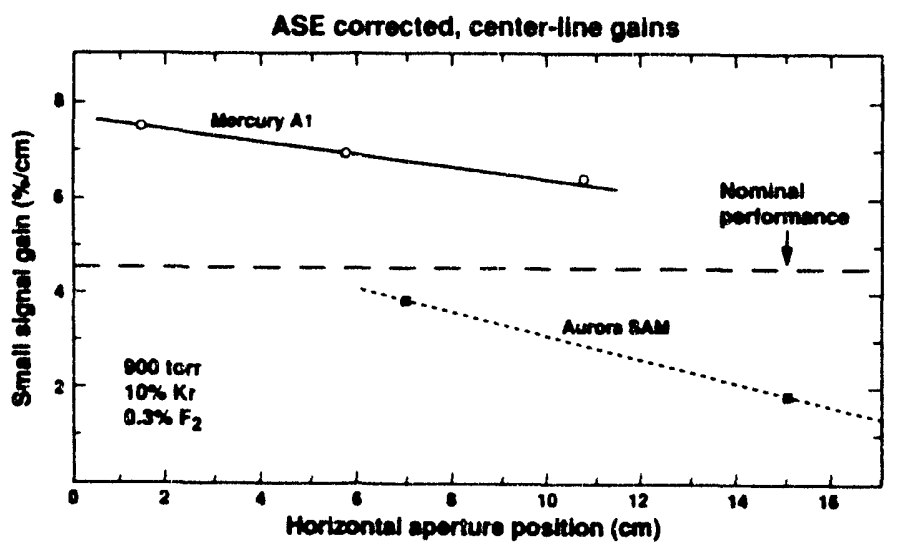

Figure 2. The laser-extracted volume is moved closer to the diode foil and a kilogauss guide field is added. This increases the laser pumping significantly and reduces spatial inhomogeneity.
We modified the foil support structure and added a guide magnetic field to SAM. The upper curve of Figure 2 indicates considerably higher small-signal gain, by a factor of two to four times, and only $20 \%$ variation across the extracted region. The small-signal gain is everywhere above the $4.5 \% \mathrm{~cm}^{-1}$ required.

\section{Modifications to Plute (Amplifier 2)}

The second amplifier. Pluto, was constructed by modifying Aurora's fourth amplifier, Large Aperture Module (LAM) [8]. Figure 3 shows the modified LAM diode.

LAM's pulsed power architecture was a set of two Marx generators that each charged two PFLs in parallel. The PFLs were discharged into two opposing diodes by trigatron switches. Divertors were provided to discharge the PFLs into matched resistors when the output switches failed to fire.
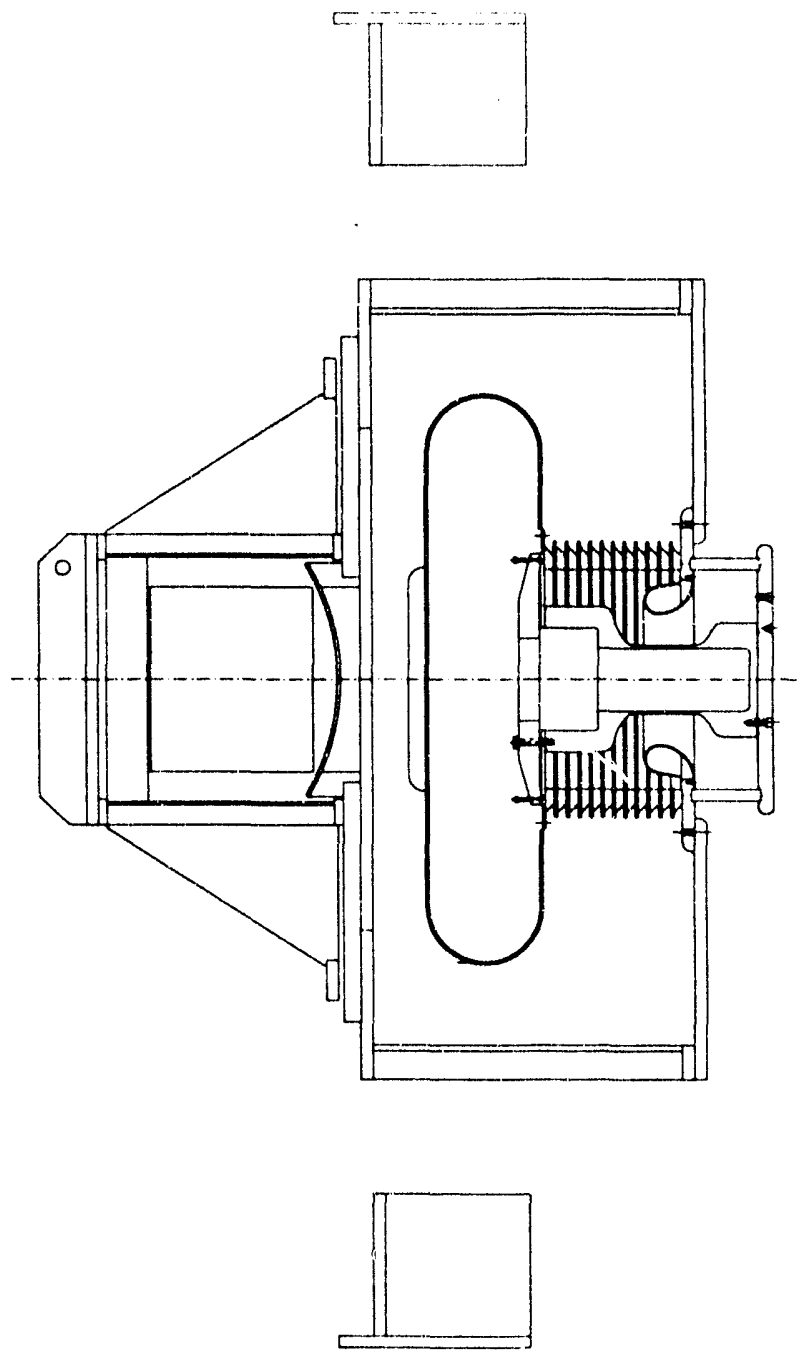

Figure 3. The diode of Pluto has a $40-\mathrm{cm}$ high by $200-\mathrm{cm}$ long emitter, rotated two degrees to counteract electron-beam twisting. The laser chamber has a $40-\mathrm{cm}$ square aperture. The stacked-ring high-voltage bushing is oil-filled. One of two guide field magnets is shown. The advanced-design foil support structure is depicted.

\section{Aperiure Reduction}

The laser aperture was reduced from $100 \mathrm{~cm}$ square to $40 \mathrm{~cm}$ square. The emitter height was also reduced from $100 \mathrm{~cm}$ to $40 \mathrm{~cm}$. The reduced aperture size requires considerably smaller and cheaper fused silica windows on the laser chamber and allows single-sided pumping (see below). 
The fabrication of the Pluto laser chamber was facilitated by the existence of a $40-\mathrm{cm}$ aperture laser chamber from the Aurora Intermediate Amplifier (third amplifier). The chamber was designed for a $3-\mathrm{m}$ electron beam. We cut out the center of the stainless steel chamber and rewelded it for use with the $2-m$ electron beam of Pluto.

A $10-\mathrm{cm}$ thick adapter plate was used on LAM to attach the laser chamber to the diode chamber. A thinner 4-cm adapter plate is used on Pluto, reducing the drift region in the diode by $6 \mathrm{~cm}$.

\section{Pulsed Power Reduction}

Calculation of laser pumping and extraction indicate that $35 \%$ higher small-signal gain is expected at 600 torr than at 900 torr. [105 kW/cc specific pump power and 10\%/90\% krypton/argon mixture were used in the calculations.] Operation at 600 torr allows single-sided pumping with good spatial uniformity across the $40-\mathrm{cm}$ laser extraction region.

Single-sided pumping on Pluto, compared with double-sided pumping on LAM, reduces the major pulsed power components by a factor of two and improves diagnostic and maintenance access to the diode and laser chambers. Only one Marx generator, one pair of pulse forming lines (PFLs), and one diode are required for Mercury. The reduction of components increases reliability.

Electrical stress parameters were also reduced, with the exception of diode current density. The water-filled, stainless steel PFLs were reduced in length by $25 \%$, from $10.8 \mathrm{~m}$ to $8.0 \mathrm{~m}$. The decision to use a 240 -ns laser pulse train on the Mercury system, rather than the 480-ns pulse train of Aurora lcu to the reduction in PFL length. (One meter of PFL equals $60 \mathrm{~ns}$ of diode operation.) The required diode voltage is $550 \mathrm{kV}$ on Pluto, versus $700 \mathrm{kV}$ on LAM, a reduction of $20 \%$. The shorter pulse duration, coupled with lower voltage, promises to increase reliability through stress reduction, particularly on bushings and switches. These were the high failure rate components of the Aurora pulsed power system. Charge transfer through the Pluto switches is only 60\% of LAM.

Further improvements in reliability could be achieved by reducing the risetime of the diode, which would allow further PFL reduction, and by diverting energy from the PFLs to a resistive load after the pulse, which would prevent afterpulse ringing.

\section{Diode Modification}

Diode current density is increased by a factor of two, from $25 \mathrm{~A}$ $\mathrm{cm}^{-2}$ on LAM's $100-\mathrm{cm}$ by $200-\mathrm{cm}$ emitter to $50 \mathrm{~A} \mathrm{~cm}^{-2}$ on Pluto's $40-\mathrm{cm}$ by $200-\mathrm{cm}$ emitter. The anode-cathode gap was reduced from $8 \mathrm{~cm}$ on LAM to $5 \mathrm{~cm}$ on Pluto to maintain $1.5-\Omega$ impedance. For cold-cathode diodes, impedance varies like $A^{-1} \mathrm{~V}^{-0.5 D^{-2}}$ (Area, Voltage, a-k gap Distance) [9].

A thin foil was installed on the back of the foil support structure. This foil serves as anode and as an absorber for low-energy electrons in the electron beam afterpulse. We have used such a prefoil on SAM and Charon and found that it prolongs the life of the pressure foil. In addition, we have seen evidence that wire anodes are irraged through the pressure foil and into the laser gas [10]. The refoil avoids this inhomogeneity and may induce less transve: seating of the beam.

The electron beam is distorted when traveling from cathode to laser chamber, because the electrons follow the twisted magnetic field lines created by the combination of guide field and self field. The beam suffers shear and rotation, as indicated by imaging on film [10]. We constructed the cathode to allow counter-rotation of the electron emitter and installed it rotated by two degrees. Imaging the beam on PERM film indicated that the technique worked.

\section{Foil Support}

Figure 4 shows the predicted performance of Pluto with $40 \%$ and $60 \%$ electron transmission from the diode through the foil support structure to the pressure foil. Figure 5 shows transmission data for a conventional planar structure [11] that incorporates a 1-mil titanium anode/prefoil. The rib structure blocks $12 \%$ of the electron beam in this design. The non-normal passage of the electron beam through the structure produces shadows, causing enhanced losses.

This experiment, without pressure foil, indicates that Pluto pumping will be adequate.

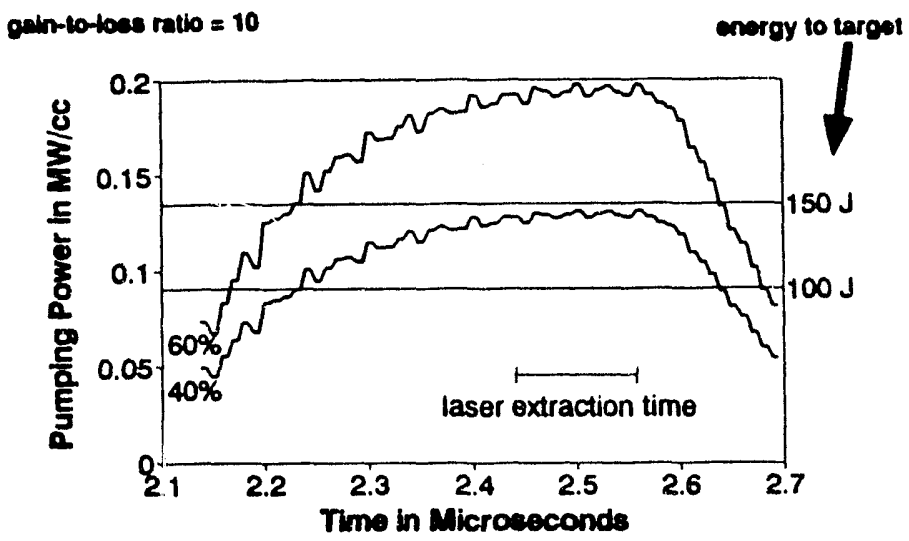

Figure 4. Model predictions indicate that $120 \mathrm{~J}$ to target will be achieved. The energy produced is sensitive to the electron transmission of the Pluto foil support structure

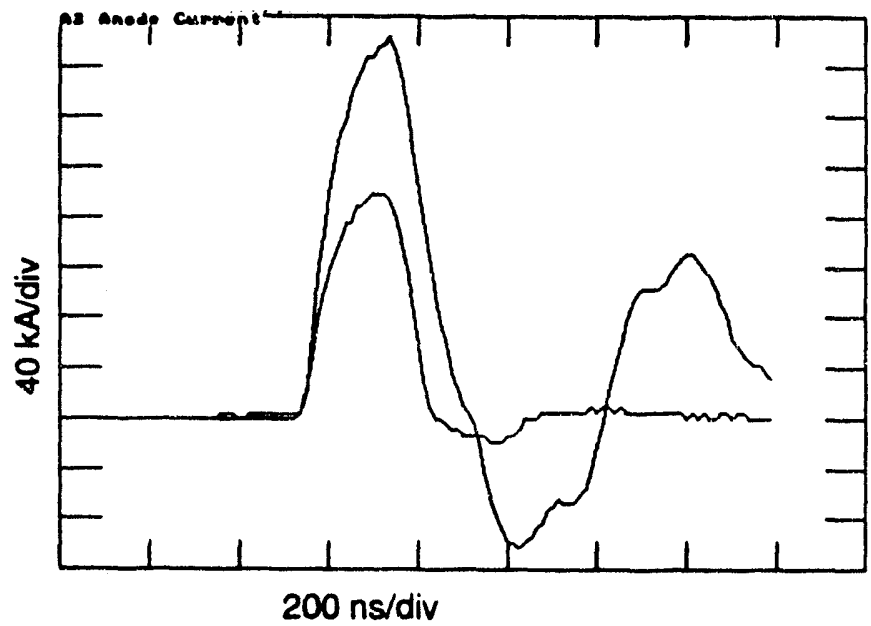

Figure 5. Measurements with the Pluto foil support and a 1-mil titanium anode/prefoil. Comparison of currents entering and leaving the support indicate $60 \%$ electron transmission.

\section{High Transpor Foil Support}

A high-transport foil support structure has been designed for Pluto. This support structure is based on a curved geometry for foil support, which takes advantage of the strength of foils and support cables when used in pure tension. It is a departure from the traditional support structures, designed with thick ribs to maintain a planar profile. A full description of this design was presented at this conference [12].

Our goal is to increase the energy transport from diode to laser gas from present values of $30 \%$ to the $50-60 \%$ range. The increase in transport efficiency will make future $\mathrm{KrF}$ amplifiers cheaper and more efficient. The IFE mission, in particular, is very sensitive to the energy efficiency of the XrF laser system. 


\section{Conclusion}

Modifications to Charon are cr.nplete and the amplifier is operational. The pump powe- exceeds requirements and spatial uniformity has been improved. Foil lifetime has been greatly enhanced, with foils now surviving undivered shots.

Modifications to Pluto are complete and the amplifier is undergoing qualification. The pulsed power system is operational. Diode tests indicate that it will meet requirements. Laser tests are imminent.

\section{Acknowledgments}

This work was supported by the United States Department of Energy through the University of California under contract W-7405-ENG-36.

The authors thank Garry Allen and Michael Kang for their significant contributions to these amplifier ciesigns.

\section{References}

[1] Stephen J. Czuchlewski, et al., "Mercury: The Los Alamos ICF KrF Laser System," Proceedings of the International Conference on Lasers '92, (1993).

[2] Louis A. Rosocha. et al., "Aurora Multikilojoule $\mathrm{KrF}$ Laser System Prototype for Inertial Confinement Fusion," Fusion Technology, 11, 497 (1987).

[3] Johnny E. Jones, et al., "Performance of the Aurora KrF ICF Laser System," Proceedings of the Intermational Conference on Lasers '89, p.88, (1990).

[4] George W. York, et al., "Mercury Laser Facility for Inertial Confinement Fusion - Phase I: Version 2.01," LA-UR 921593, 14 August 1992.

[5] Evan A. Rose, et al., "Pulsed Power Considerations for Electron Beam Pumped Krypton Fluoride Lasers for Inertial Confinement Fusion Applications," SPIE International Symposium on High Power Lasers, Los Angeles, CA, January, 1991.

[6] Evan A. Rose, et al., "A Megajoule Class Krypton Fluoride Amplifier for Single Shot, High Gain ICF Application," Fusion Technology, 15, 364, (1989).

[7] David B. Harris, et al., "Improved KrF Laser Design for the Laboratory Microfusion Facility," Proceedings of the IEEE/NPSS Symposium on Fusion Engineering, San Diego, CA, September, 1991.

[8] Evan Rose and David Hanson, "Final Amplifier Design and Mercury," Proceedings of the Intemational Conference on Lasers '91, p.701, (1992).

[9] John J. Coogan, Evan A. Rose, Roger P. Shurter: "Optical and Electrical Investigations into Cathode Ignition and Diode Closure," 8th IEEE Intemational Pulsed Power Conference Proceedings, p.559, (1991).

[10] Charles R. Mansfield, Hain Oona, and Roger P. Shurter, "Beam Rotation and Shear in a Large Electron Beam Diode," IEEE Transactions on Plasma Science, 19, 840, (1991).

[11] Roger P. Shurter, et al., "Performance Improvements with Advanced Design Foils in High-Current Electron Beam Diodes," IEEE Transactions on Plasma Science, 19, 846, (1991).

[12] John P. Brucker and Evan A. Rose, "Foil Support Structures for Large Area Diodes," 9th IEEE International Pulsed Power Conference Proceedings, (1993). 

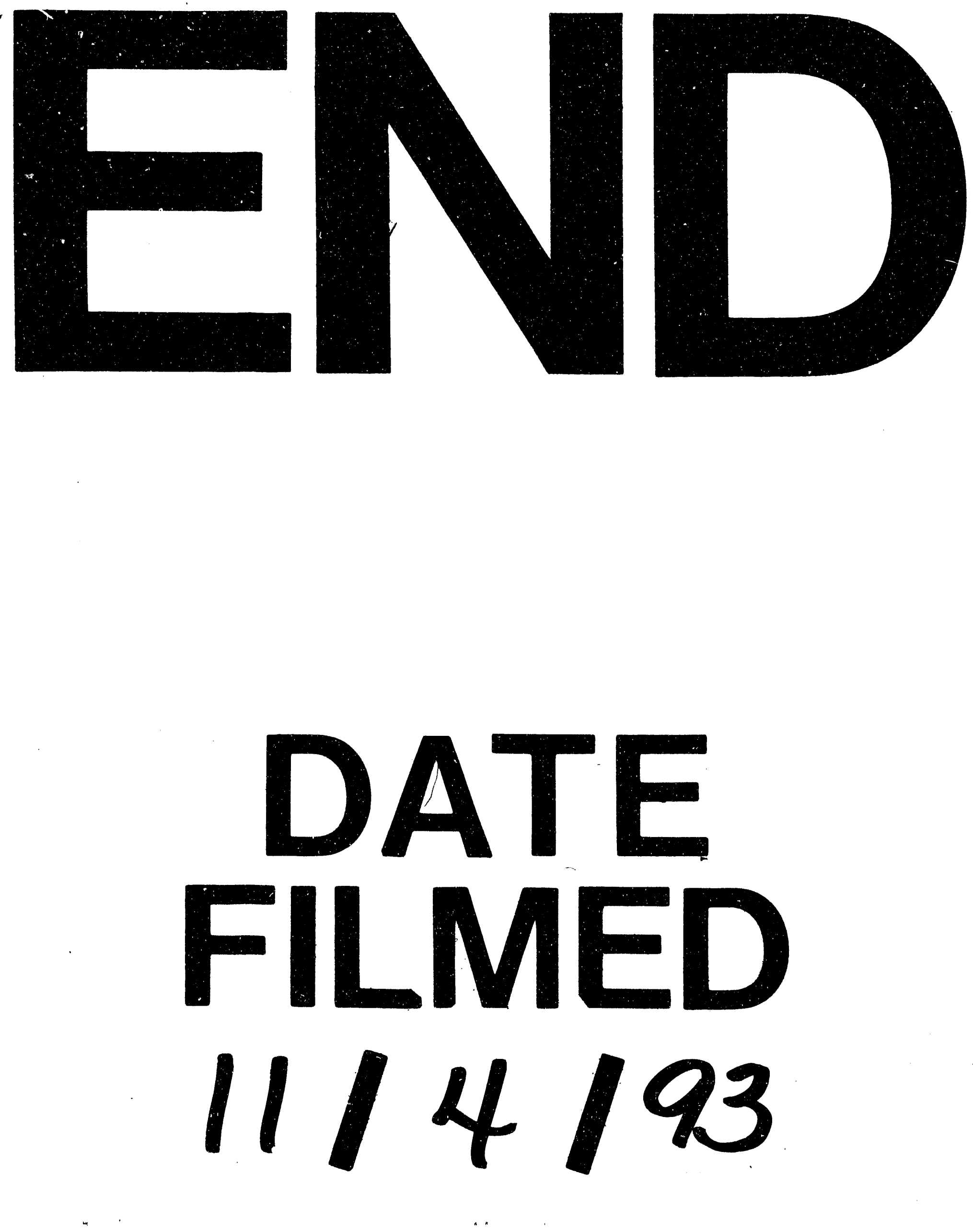
